\title{
Fatores determinantes do uso de instrumentos de gestão de risco de preço por pecuaristas de corte do Estado de São Paulo
}

\author{
Factors influencing beef cattle farmers use of risk management instruments in the State of São Paulo, \\ Brazil
}

\author{
Marcelo José Carrer $^{\text {I* }}$ Rodrigo Lanna Franco da Silveira' $^{\mathrm{II}}$ Hildo Meirelles de Souza Filho \\ Marcela de Mello Brandão Vinholis ${ }^{\text {III }}$
}

RESUMO

A oscilação não favorável nos preços do boi gordo se constitui em um dos principais riscos da atividade pecuária. De forma a gerenciar tal risco, contratos a termo e futuros podem ser utilizados por pecuaristas. No entanto, o uso desses derivativos é bastante restrito, sendo as razões baseadas nas características do produtor e de seu negócio. Nesse contexto, o presente estudo teve o objetivo de identificar os determinantes da adoção de mecanismos de gestão de risco de preço do boi gordo por pecuaristas de corte no Estado de São Paulo. Para atingir esse objetivo, foram levantados dados primários junto a uma amostra de 86 pecuaristas, sendo as respostas analisadas por meio de um modelo Logit. Os resultados apontaram que, quanto maior a receita do pecuarista e seu grau de intensidade tecnológica e quanto menor o uso de dívidas para custeio e investimento, maior a probabilidade de se usar algum mecanismo de proteção.

Palavras-chave: gestão de risco, pecuária de corte, contratos futuros e contratos a termo.

\section{ABSTRACT}

The beef cattle price volatility is one of the main risks of this activity. To manage this risk, forward and futures contracts can be used by beef cattle farmers. However, the use of these contracts is quite restricted. The reasons are based on the characteristics of farmers and his business. In this context, this study aimed to identify the determinants factors of adoption of risk management mechanisms by beef cattle farmers in the State of São Paulo, Brazil. A logit model was used to analyze data from a sample of 86 beef cattle farmers. The results showed that the factors that significantly affected the adoption of the risk management tools were farm income, technological intensity and business leverage.

Key words: risk management, beef cattle, futures contract and forward contract.

\section{INTRODUÇÃO}

A produção rural é cercada de especificidades, das quais se destacam a dependência do clima, o longo período de maturação dos investimentos e o ciclo de desenvolvimento dos produtos rurais, marcado por períodos de safra e entressafra. A partir de tais características, dois tipos de riscos emergem, possuindo significativa relevância em termos de impacto na receita e, consequentemente, na viabilidade econômica da atividade rural: i) o risco de perdas na produção por conta de eventos climáticos; e ii) o risco de variações nos preços dos produtos. $\mathrm{O}$ primeiro risco pode ser minimizado com a utilização mais eficiente dos fatores de produção e com a adoção do seguro rural pelos produtores. Já o risco de variações nas cotações pode ser minimizado com um melhor planejamento do processo de comercialização e com a adoção de mecanismos de proteção (hedge).

Diferentes instrumentos podem ser utilizados pelos produtores rurais brasileiros para

'Departamento de Engenharia de Produção, Universidade Federal de São Carlos (UFSCar), São Carlos, SP, Brasil. E-mail: marcelojcarrer@dep.ufscar.br.*Autor para correspondência.

IInstituto de Economia, Universidade Estadual de Campinas (UNICAMP), Campinas, SP, Brasil.

"IEmbrapa Pecuária Sudeste, São Carlos, SP, Brasil. 
gerenciamento de variações nos preços dos produtos agropecuários. Pelo seu maior uso entre os produtores, destacam-se os contratos a termo e os futuros.

O primeiro se caracteriza por ser negociado em mercado de balcão, em que, a partir de cláusulas customizadas, o produtor rural se compromete a entregar uma quantidade pré-especificada da commodity para a agroindústria em uma data futura a um preço préestabelecido no momento do acordo. Assim como o termo, o contrato futuro também estabelece um acordo de compra ou venda de um ativo a um preço préestabelecido para entrega em data futura. No entanto, tal contrato é padronizado e negociado em bolsa de mercadorias e futuros. A padronização permite a reversão da posição a qualquer instante, fato que não é possível no termo, dada a sua customização. Nesse aspecto, cabe mencionar que grande parte das negociações no mercado futuro não é liquidada mediante entrega física. O produtor rural, em geral, encerra sua posição antes do vencimento do contrato, obtendo proteção contra variações não desejadas nos preços e, simultaneamente, transaciona seu produto no mercado a vista com as agroindústrias e tradings ao preço vigente. Vale ainda observar que a bolsa, de forma a gerenciar o risco de crédito da transação, estabelece dois mecanismos fundamentais para o bom funcionamento dos mercados futuros: o ajuste diário e a margem de garantia. $\mathrm{O}$ mecanismo de ajuste diário estabelece que os agentes participantes ajustem diariamente sua posição financeira conforme a oscilação dos preços futuros. Dessa forma, cria-se um fluxo financeiro de pagamentos e recebimentos durante a vigência do contrato, não existindo, portanto, um único acerto financeiro na data de expiração do derivativo (como é comum no mercado a termo). $\mathrm{O}$ segundo mecanismo, a margem de garantia, consiste em um depósito de certo montante, definido pela Câmara de Compensação da bolsa, que deve ser feito pelos agentes participantes no dia útil seguinte à negociação. Caso exista um evento de inadimplemento relativo ao ajuste diário, a bolsa utiliza a margem de garantia para o pagamento.

Estudos têm analisado os fatores que levam à adoção de técnicas de gestão do risco de preço na agropecuária (SHAPIRO \& BRORSEN, 1988; ASPLUND et al., 1989; MAKUS et al., 1990; TURVEY \& BARKER, 1990; GOODWIN \& SCHROEDER, 1994; PENNINGS \& LEUTHOLD, 2000; VELANDIA et al., 2009; MARQUES \& AGUIAR, 2004; SILVEIRA et al., 2011). Estes dois últimos foram desenvolvidos a partir de pesquisas com sojicultores e cafeicultores nacionais, respectivamente.
Em geral, dois grupos de variáveis são identificados para explicação do uso de instrumentos de gestão de risco de preço. O primeiro tem base nas características do produtor, tais como: escolaridade, experiência na atividade, idade, se membro de uma cooperativa, participação em cursos/treinamento, grau ao qual se mantém informado sobre o mercado, nível de conhecimento sobre derivativos, preferências relativas ao modelo de administração da atividade e aspectos de seu comportamento, como, por exemplo, excesso de confiança na gestão administrativa e propensão ao risco. O segundo grupo consiste nas características da propriedade e do negócio, incluindose: tamanho da produção, renda da atividade específica, renda em outras atividades, alavancagem financeira, diversificação da produção, existência de seguro rural e participação em programas do governo de proteção contra queda de preços.

A pecuária de corte no Estado de São Paulo é caracterizada por significativas variações nos preços da arroba paga ao produtor rural e por conflitos no processo de comercialização entre pecuaristas e frigoríficos (SOUZA FILHO et al., 2010). Diante desse cenário, é de fundamental importância para os pecuaristas a adoção de mecanismos de gestão do risco das variações nos preços do boi gordo. Além de proteger os pecuaristas de eventuais oscilações não favoráveis nas cotações e possibilitar um melhor planejamento da atividade, alguns mecanismos podem ainda trazer externalidade positiva no que tange ao desenvolvimento de relações mais estáveis entre pecuaristas e frigoríficos, minimizando os conflitos existentes nessa cadeia produtiva e aumentando a eficiência sistêmica da cadeia. Ademais, observa-se que o uso de instrumentos de gestão de risco de preço garante um maior acesso às linhas de crédito junto às instituições financeiras (CARRER, 2012).

Nesse contexto, o presente estudo tem o objetivo de identificar os determinantes da adoção de mecanismos de gestão de risco de preço do boi gordo por pecuaristas de corte no Estado de São Paulo. O estudo ganha relevância, pois, apesar da importância dos instrumentos de administração de risco acima citados, não se observam estudos dessa temática envolvendo este segmento produtivo.

\section{MATERIAL E MÉTODOS}

Amostra

Os dados utilizados no presente estudo tiveram base em uma pesquisa de campo, na qual foram entrevistados 86 pecuaristas de corte no Estado de São Paulo. Estes detinham 189 propriedades rurais 
localizadas no Estado. As entrevistas foram presenciais, ocorrendo de forma estruturada, sendo realizadas no período de janeiro a setembro de 2011. Os dados coletados referem-se ao ano de 2010.

Os pecuaristas, que fizeram parte da amostra deste estudo, localizavam-se em 10 mesorregiões de maior representatividade na produção de bovinos de corte do Estado de São Paulo. A distribuição dos 86 pecuaristas se deu nas seguintes mesorregiões: 19 em Araçatuba, 17 em Presidente Prudente, 14 em Ribeirão Preto, 13 em Bauru, 13 em São José do Rio Preto e 10 em outras mesorregiões. Procurou-se obter uma amostra que representasse os mais diversos sistemas de produção (confinamentos, semi-confinamentos, pasto extensivo, etc.), as diferentes características presentes entre os produtores e os diferentes mecanismos de comercialização adotados pelos produtores (mercado spot, mercados futuros, contratos a termo, etc.).

Modelos de escolha qualitativa

Para identificar os fatores que determinam a adoção de mecanismos de gestão de risco (contrato a termo e/ou contratos futuros) por produtores de bovinos de corte no Estado de São Paulo, utilizou-se o modelo Logit.

Ao tomar a decisão de adotar ou não adotar tais mecanismos, pode-se pressupor que o produtor considera as vantagens e desvantagens marginais da utilização dos mecanismos de gestão de risco. Como os parâmetros dessa decisão não são geralmente observáveis, para cada propriedade rural $i$, pode ser definida uma variável latente, $y^{*}$, como:

$$
y_{i}^{*}=\beta^{\prime} X_{i}+u_{i} i=1, \ldots, N
$$

Em que $X$ denota um conjunto de variáveis explicativas. O padrão observado de adoção de mecanismos de gestão de risco pode ser descrito por uma variável dummy, $y$, tal que $y_{i}=1$ se o produtor $i$ adota mecanismos de gestão de risco e $y_{i}=0$ se não adota tais mecanismos. Os valores observados de $y$ são relacionados com $y^{*}$ da seguinte forma:

$$
\begin{aligned}
y_{i}=1 \text { se } y_{i}^{*}>0 \\
y_{i}=0 \text { caso contrário e } \\
\operatorname{Pr}\left(y_{i}=1\right)=\operatorname{Pr}\left(y_{i}^{*}>0\right)=\operatorname{Pr}\left(u_{i}>-\beta^{\prime} X_{i}\right)=1-F\left(-\beta^{\prime} X_{i}\right) \\
=F\left(\beta^{\prime} X_{i}\right)
\end{aligned}
$$

Em que $F$ é uma função de distribuição cumulativa para $u$ e uma distribuição simétrica é assumida. Utilizando procedimentos de máxima verossimilhança, as estimativas dos parâmetros $\beta$ são obtidas. No modelo Logit, uma função de distribuição cumulativa logística é considerada:

$$
\begin{aligned}
\operatorname{Pr}\left(\mathrm{y}_{i}\right. & =1)=\frac{e^{\beta X}}{1+e^{\beta X}} \\
& =\Lambda\left(\beta^{\prime} X\right)
\end{aligned}
$$

Em que X denota a função de distribuição cumulativa logística.

Como mencionado na introdução deste trabalho, os fatores determinantes da adoção de mecanismos de gestão de risco evolvem, em geral, as características do produtor e de seu negócio. Nesse sentido, foram construídas oito variáveis para explicar a decisão de uso ou não de contratos a termo e futuros no gerenciamento do risco de preço da pecuária: idade, escolaridade, existência de renda em outra atividade não rural, grau ao qual se mantém informado sobre o mercado, inserção em redes políticas, receita com a venda de bovinos para abate, grau de alavancagem do negócio e grau de intensidade em tecnologia no sistema de produção. A tabela 1 apresenta tais variáveis, caracterizando-as.

Cabe realizar algumas observações no que diz respeito às variáveis do modelo. A amostra é constituída por produtores com alta escolaridade. Dessa forma, para tal variável, optou-se por utilizar a realização de curso de pós-graduação para distinguir os pecuaristas neste quesito. Em relação à variável "participação em redes políticas", para obtê-la, consideraram-se aqueles produtores que participam de associações de classe e/ou cooperativas e/ou sindicatos rurais e/ou redes informais para compra de insumos ou venda do gado. Cabe ainda destacar que, neste artigo, definem-se redes políticas como instituições informais que se baseiam em regras acordadas para chegar a um objetivo comum, ou seja, institucionalizando um mecanismo de coordenação horizontal e reduzindo assim os custos de informação e transação, criando confiança e reduzindo incertezas (BÖRZEL, 1997). Quanto à renda em outras atividades não rurais, enfatizou-se a renda em atividades fora da agricultura, pois a grande maioria dos produtores possuía outra atividade agrícola. Por fim, a variável "grau de intensidade tecnológica" foi desenvolvida de acordo com o uso ou não de confinamento na engorda dos animais. Justifica-se essa escolha com estudo da EMBRAPA(2005), o qual classifica o sistema de confinamento como o mais intensivo em capital na pecuária de corte brasileira. Por hipótese, estabeleceuse que um pecuarista com maior intensidade tecnológica é aquele que adota confinamento como sistema de produção para engordar os animais.

A partir de tais dados, o modelo Logit foi utilizado a fim de obter as relações entre uso de 
Tabela 1 - Variáveis utilizadas para identificar os fatores que determinam a adoção de mecanismos de gestão de risco de preço pelos pecuaristas de corte entrevistados.

\begin{tabular}{|c|c|}
\hline Variável & Descrição \\
\hline \multicolumn{2}{|l|}{ Variável dependente } \\
\hline $\begin{array}{l}\text { Uso de contratos futuros e/ou termo para gestão do risco } \\
\text { de preço (GEST) }\end{array}$ & Variável dummy de valor igual a 1 se utiliza e 0 caso contrário. \\
\hline \multicolumn{2}{|l|}{ Variáveis explicativas } \\
\hline Idade (ID) & Idade do produtor. \\
\hline Escolaridade (ESC) & Variável dummy de valor igual a 1 se tem pós-graduação e 0 caso contrário. \\
\hline Participação em redes políticas (RP) & $\begin{array}{l}\text { Variável dummy de valor igual a } 1 \text { se participa de redes políticas e } 0 \text { caso } \\
\text { contrário. }\end{array}$ \\
\hline Renda em outras atividades não rurais (ROT) & $\begin{array}{l}\text { Variável dummy de valor igual a } 1 \text { se possuía outras rendas além da } \\
\text { atividade rural e } 0 \text { caso contrário. }\end{array}$ \\
\hline $\begin{array}{l}\text { Acompanhamento do mercado em tempo real (on-line) } \\
\text { (ACOMP) }\end{array}$ & $\begin{array}{l}\text { Variável dummy de valor igual a } 1 \text { se acompanha o mercado pela internet e } \\
0 \text { caso contrário. }\end{array}$ \\
\hline Grau de alavancagem financeira (ALAV) & Percentual do custeio e investimento financiados com capital de terceiros. \\
\hline Grau de intensidade tecnológica (IT) & $\begin{array}{l}\text { Variável dummy de valor igual a } 1 \text { se tem alto grau tecnológico no sistema } \\
\text { de produção e } 0 \text { caso contrário. }\end{array}$ \\
\hline Receita bruta da produção (REC) & Receita bruta oriunda da venda de animais para abate. \\
\hline
\end{tabular}

contratos a termo/futuros e as variáveis acima descritas. Por hipótese, espera-se uma relação positiva entre uso de contratos futuros e escolaridade, participação em redes políticas, grau de alavancagem financeira, tamanho de produção e acompanhamento do mercado. Adicionalmente, relação inversa é esperada para as variáveis: idade e renda em outras atividades não rurais. Por hipótese, considera-se que a idade do produtor é proporcional ao modelo conservador de gestão. Quanto à variável renda em outra atividade, assume-se que a diversificação de negócios já se traduz em uma técnica de proteção contra riscos de preços.

\section{RESULTADOS E DISCUSSÃO}

Dentre os 86 pecuaristas, 31 deles, o que corresponde a aproximadamente $36 \%$ da amostra, adotaram algum mecanismo de gestão de risco de preço no ano de 2010. Os demais, 55 pecuaristas, não utilizaram nenhum mecanismo de gerenciamento de risco.

Os pecuaristas que adotaram mecanismos de gestão de risco de preço (grupo 1) apresentaram, em média, uma idade pouco inferior em relação àqueles que não negociavam contratos a termo/futuros (grupo 2) média de 49 anos para os produtores do grupo 1 e 53 anos para os produtores do grupo 2 . No que tange à escolaridade dos pecuaristas que gerenciaram risco de preço, 39\% detinham pós-graduação, sendo que no grupo 2 somente $18 \%$ possuíam tal formação. A participação em redes políticas, por sua vez, apresentou-se bastante elevada na amostra do estudo. Uma parcela de $87 \%$ dos produtores do grupo 1 participava de redes políticas - já no outro conjunto de pecuaristas, tal proporção foi de $71 \%$. No que tange à presença de renda fora $\mathrm{da}(\mathrm{s})$ propriedade(s) rural(is), no grupo $1,65 \%$ tinham tal característica, enquanto que, no grupo 2 , tal índice foi de 55\%. Por fim, ao analisar o grau de acompanhamento do mercado pela internet, verificou-se que $65 \%$ dos produtores que gerenciavam risco de preço acompanhavam o mercado em tempo real pela internet. Dentre os produtores do grupo 2, apenas $24 \%$ possuíam tal característica.

Com relação às características do negócio, para o grupo que gerenciava risco de preço, os custos 
de produção e investimentos eram financiados majoritariamente por recursos próprios - média de $77 \%$ de recursos de próprios e $23 \%$ de terceiros. A mesma situação foi observada no segundo grupo, existindo menor utilização de capital de terceiros - média de 83\% de recursos de próprios e 17\% de terceiros. Em relação à intensidade tecnológica no sistema de produção dos pecuaristas do grupo $1,90 \%$ adotavam confinamento como sistema de produção na engorda dos animais. Para o grupo 2, apenas $24 \%$ realizavam confinamento. Quanto à receita bruta, houve uma diferença considerável nas médias dos dois grupos. Contudo, deve-se tomar cuidado ao interpretar essa variável, devido ao alto desvio-padrão existente, principalmente para os pecuaristas que adotavam gestão de risco de preços (desvio padrão de $\mathrm{R} \$ 24.908 .472,30$ para o grupo 1 e $R \$ 1.247 .231,97$ para o grupo 2). No grupo 1, a receita média oriunda da venda de animais para abate em 2010 foi de aproximadamente $R \$ 17,85$ milhões, enquanto que, no grupo 2, tal média foi de $\mathrm{R} \$ 1,16$ milhão. Analisando a mediana dessa variável, no grupo 1, o valor foi de R \$ 7.554.762,00 e, no grupo 2, essa medida atingiu $\mathrm{R} \$ 561.600,00$, confirmando a significativa diferença na receita da produção para os dois grupos de produtores.

A tabela 2 apresenta os resultados obtidos a partir da estimação do modelo Logit. Das oito variáveis explicativas utilizadas no modelo, três se mostraram significativas, sendo elas: receita, intensidade tecnológica e grau de alavancagem.

Constatou-se que, quanto maior a escala de produção e, por consequência, a receita do pecuarista, maior a probabilidade de se usar algum mecanismo de proteção. Esse resultado foi similar ao obtido por SHAPIRO \& BRORSEN (1988), ASPLUND et al. (1989), MAKUS etal.(1990), GOODWIN\& SCHROEDER (1994), MARQUES \& AGUIAR (2004), SILVEIRAet al. (2011).

Adicionalmente, observou-se que, quanto maior o grau de intensidade tecnológica (medido pela adoção de confinamento), maior a propensão de se usar instrumentos de gerenciamento do risco de preço. GOODWIN \& SCHROEDER (1994) obtiveram resultado semelhante ao utilizar o grau de uso de insumos. Conclui-se, assim, que, quanto mais preocupado com sua produtividade for o pecuarista, maior a probabilidade de se usar contratos a termo ou futuros. Ademais, quando o pecuarista adota o confinamento como sistema de produção, a liquidez da atividade se reduz, principalmente se comparado àqueles que adotam o sistema de pasto extensivo. No caso do confinamento, os animais devem ser comercializados assim que atingirem o peso ótimo (presença de especificidade temporal), e cada dia que o animal permanece no confinamento após atingir o peso ótimo

Tabela 2 - Coeficientes estimados do modelo logit para análise dos determinantes do uso de mecanismos de gestão de risco de preço por pecuaristas de corte entrevistados.

\begin{tabular}{llll}
\hline Variável & Coeficiente & Valor de Z & p-valor \\
\hline C & $-3,024228$ & 2,024527 & 0,1352 \\
ID & $-0,031519$ & 0,037451 & 0,4000 \\
ESC & 0,015389 & 0,956024 & 0,9872 \\
RP & 1,004461 & 0,959568 & 0,2952 \\
ROT & 0,586808 & 0,876696 & 0,5033 \\
ACOMP & 0,959054 & 0,810991 & 0,2370 \\
ALAV & $-4,303035$ & 2,562509 & $0,0931^{* * *}$ \\
IT & 3,055884 & 0,914905 & $0,0008^{*}$ \\
REC & $5,73 \mathrm{E}-07$ & $2,38 \mathrm{E}-07$ & $0,0160^{* *}$ \\
Log da verossimilhança & $-23,27$ & & \\
Estatística LR (8gl) & 65,89 & & \\
p-valor (estatística LR) & 0,00 & & \\
R'McFadden & 0,5860 & & \\
\hline
\end{tabular}

Fonte: Resultados da pesquisa.

${ }^{*}$ Significativo a $1 \%,{ }^{* *}$ Significativo a $5 \%,{ }^{* * *}$ Significativo a $10 \%$.

* Idade (ID), escolaridade (ESC), participação em redes políticas (RP), renda em outras atividades não rurais (ROT), acompanhamento do mercado em tempo real (ACOMP), grau de alavancagem financeira (ALAV), grau de intensidade tecnológica (IT), receita bruta da produção (REC). 
representa um alto custo para o pecuarista. Logo, o pecuarista precisa planejar de forma mais eficiente a comercialização dos animais, protegendo-se das variações nas cotações e programando a venda de forma antecipada, conforme constatado nos resultados do presente estudo.

Já em relação à variável grau de alavancagem, o sinal obtido foi contrário ao esperado. Como verificado em boa parte dos artigos do tema, espera-se que um produtor que já tenha alto risco financeiro utilize mecanismos de proteção de forma a garantir o pagamento de suas dívidas. No entanto, os resultados apontaram para uma relação inversa. Na amostra utilizada, quanto menor o uso de dívidas para custeio e investimento, maior a probabilidade de se adotar o hedge em mercados a termo ou futuros. Uma possível explicação para este resultado pode estar associada ao fato de os produtores, que adotavam mecanismos de gestão de risco, deterem menor propensão ao risco financeiro. Desse fato, explica-se que esses produtores preferem utilizar o capital próprio para financiar a atividade, assim como adotar mecanismos de trava dos preços. Como a amostra deste estudo possui um grande número de produtores capitalizados, estes de fato não precisam recorrer a recursos de terceiros para financiar a atividade, o que explica o resultado contrário ao esperado para tal variável.

Por fim, vale observar que o teste de máxima verossimilhança indicou que as variáveis explicaram de forma satisfatória a variável dependente "uso de contratos futuros ou a termo para gestão do risco de preço".

\section{CONCLUSÃO}

Este trabalho teve o objetivo de identificar os fatores que levam os produtores de bovinos de corte no Estado de São Paulo a adotar mecanismos de gestão de risco de preço. Verificou-se que os produtores com maior receita na produção, maior intensidade em tecnologia no sistema de produção e menor alavancagem financeira possuem maior probabilidade de adotar contrato a termo e/ou contratos futuros.

Os resultados do estudo podem ser utilizados tanto pelos agentes que ofertam os mecanismos de gestão de risco para os produtores de bovinos de corte em São Paulo, como também pelo governo, de forma a incentivar a difusão de tais mecanismos. Para o produtor, a gestão do risco de preço colabora para um melhor planejamento dos investimentos na produção rural, visto que o retorno financeiro da atividade fica pré-estabelecido.

Por fim, cabe ressaltar que o presente estudo apresenta caráter inovador para esse segmento produtivo. Apesar da importância do gerenciamento de risco de preços na pecuária e da grande oscilação observada nos preços do boi gordo no Brasil, não foram encontrados outros estudos que objetivaram identificar os determinantes do uso de mecanismos de gestão de risco entre pecuaristas de corte.

\section{AGRADECIMENTO}

Os autores agradecem à FAPESP (Fundação de Amparo à Pesquisa do Estado de São Paulo), pelo financiamento do projeto por meio de Auxílio Regular à Pesquisa.

\section{COMITÊ DE ÉTICA E BIOSSEGURANÇA}

Os assumem inteira responsabilidade pelo fato de o
referido

\section{REFERÊNCIAS}

ASPLUND, N.M. et al. Farmers' use of forward contracting and hedging. Review of Futures Markets, v.8, p.24-37, 1989.

BÖRZEL, T. ¿Qué tienen de especial los policy networks? Explorando el concepto y su utilidad para el estudio de la gobernación europea, 1997. Disponível em: <http://revista-redes.rediris.es/ webredes/textos/policynet.doc>. Acesso em: 12 mar. 2012.

CARRER, M.J. Determinantes da demanda e da utilização de crédito rural por produtores de bovinos de corte no Estado de São Paulo. 2012. 135f. Dissertação (Mestrado em Engenharia de Produção) - Programa de Pós-graduação em Engenharia de Produção, Universidade Federal de São Carlos, SP.

EMPRESA BRASILEIRA DE PESQUISA AGROPECUÁRIA EMBRAPA. Sistemas de produção de gado de corte no Brasil: uma descrição com ênfase no regime alimentar e no abate. Documentos/EMBRAPA Gado de Corte, 2005. 40p. Disponível em: <http://www.cnpgc.embrapa.br/publicacoes/doc/ doc_pdf/doc151.pdf>. Acesso em: 05 dez. 2011.

GOODWIN, B.K.; SCHROEDER, T.C. Human capital, producer education programs, and the adoption of forward-pricing methods. American Journal of Agricultural Economics, v.76, n.4, p.936-947, 1994.

MAKUS, L.D. et al. Factors influencing farm level use of futures and options in commodity marketing. Agribusiness, v.6, n.6, p.621-631, 1990.

MARQUES, R.H.S.; AGUIAR, D.R.D. Determinantes do uso de mercados futuros pelos produtores de soja do município de Cascavel, PR. Revista de Economia e Agronegócio, v.2, n.2, p.209-233, 2004.

PENNINGS, J.M.E.; LEUTHOLD, R.M. The role of farmer's behavioral attitudes and heterogeneity in futures contracts usage. American Journal of Agricultural Economics, v.82, n.4, p.908-919, 2000.

SHAPIRO, B.I.; BRORSEN, B.W. Factors affecting farmers' hedging decisions. North Central Journal of Agricultural Economics, v.10, p.145-153, 1988. 
SILVEIRA, R.L.F. et al. An analysis of price risk management by the producers of Arabica Coffee in Brazil. In: INTERNATIONAL AGRIBUSINESS PAA-PENSA CONFERENCE, 8., 2011, Buenos Aires, Argentina. Anais... Buenos Aires: International Agribusiness PAA-PENSA Conference, 4, 2011. (CD-ROM).

SOUZA FILHO, H.M. et al. Análise da competitividade da cadeia produtiva da carne bovina do Estado de São Paulo. Informações Econômicas, v.40, n.3, p.16-28, 2010.
TURVEY, C.G., BAKER, T.G. A farm level financial analysis of farmers' use of futures and options under alternative farm programs. American Journal of Agricultural Economics, v.72, p.946-957, 1990.

VELANDIA, M. et al. Factors affecting farmers' utilization of agricultural risk management tools: the case of crop insurance, forward contracting, and spreading sales. Journal of Agricultural and Applied Economics, v.41, n.1, p.107-123, 2009. 